\title{
Corrosividade Causada por Soluções Salinas Empregadas na Estabilização Dimensional da Madeira
}

\author{
Juarez Benigno Paes ${ }^{1}$, Pedro Lício Loiola ${ }^{2}$, Rafael Leite Braz², \\ Ricardo Jorge Klitzke ${ }^{3}$, Rogy Frigeri Tiburtino ${ }^{1}$ \\ ${ }^{1}$ Departamento de Ciências Florestais e da Madeira, Universidade Federal do Espirito Santo - UFES, \\ Jeronimo Monteiro/ES, Brasil \\ ${ }^{2}$ Programa de Pós-graduação em Engenharia Florestal, Universidade Federal do Paraná - UFPR, Curitiba/PR, Brasil \\ ${ }^{3}$ Departamento de Engenharia e Tecnologia Rural, Universidade Federal do Paraná - UFPR, Curitiba/PR, Brasil
}

\section{RESUMO}

Objetivou-se avaliar a corrosividade de soluções salinas antes e depois de sua impregnação na madeira. Soluções com $5 \%$ de concentração de cloreto de lítio, carbonato de sódio, sulfato de magnésio, sulfato de zinco e sulfato de cobre II foram mantidas durante 6 horas, a $25^{\circ} \mathrm{C}, 45^{\circ} \mathrm{C} \mathrm{e}$ $100^{\circ} \mathrm{C}$ em contato com amostras de $1,25 \mathrm{~cm} \times 0,1 \mathrm{~cm} \times 7,5 \mathrm{~cm}$ (largura $\times$ espessura $\times$ comprimento) produzidas com chapas de aço carbono antes e depois de seu contato com amostras de madeira de Corymbia torelliana e Eucalyptus cloeziana. Não se observou efeito da temperatura na corrosão das soluções puras. Depois do contato com a madeira houve incremento da corrosividade para as soluções de sulfato de zinco e de cobre II. O sulfato de cobre II ocasionou a maior perda de massa nas chapas, não sendo recomendado seu contato com superfícies metálicas, como usinas de tratamento e câmaras de secagem de madeira.

Palavras-chave: corrosão, soluções salinas, extrativos, madeira.

\section{Corrosivity Caused by Salt Solutions Employed in the Wood Dimensional Stability}

\begin{abstract}
The study aimed to evaluate the corrosivity of salt solutions after and before the impregnation in the wood. Solutions with concentration of $5 \%$ of chloride of lithium, sodium carbonate, magnesium sulphate, zinc sulphate and copper sulphate II had been kept during 6 hours, at $25 ; 45$ and $100^{\circ} \mathrm{C}$, in contact with steel plates carbon samples of $1.25 \times 0.1 \times 7.5 \mathrm{~cm}$ (width $\times$ thickness $\times$ length), before and after its contact with wood samples of Corymbia torelliana and Eucalyptus cloeziana. There was no effect of the temperature in the corrosion of the pure solutions. After the contact with woods, corrosivity was incremented with copper II and zinc sulphate solutions. Copper II sulphate caused the biggest loss of mass in plates, and its contact with metallic surfaces is not recommended as wood treatment and drying chambers.
\end{abstract}

Keywords: corrosion, salt solutions, extractives, wood. 


\section{INTRODUÇÃO}

A madeira atua como material corrosivo frente a outros produtos e materiais de construção, mas somente em condições específicas de uso. Em seu estado úmido ela possui a capacidade de causar a corrosão de pregos e parafusos empregados na sua conexão. Porém a corrosão se agrava com o tempo e a tenacidade da união pode até aumentar por causa da ferrugem (Kollmann \& Côté, 1968).

Kollmann (1959) afirmou que a madeira impregnada com soluções salinas promove a corrosão mais intensamente, na maioria dos casos, quando comparada com madeiras que não foram submetidas a nenhum tipo de tratamento. A impregnação com sulfocianeto amônico $\left(\mathrm{SCNNH}_{4}\right)$ e sulfato de cobre $\left(\mathrm{CuSO}_{4} \cdot 5 \mathrm{H}_{2} \mathrm{O}\right)$ causa grande corrosividade, enquanto soluções de cloreto de zinco $\left(\mathrm{Cl}_{2} \mathrm{Zn}\right)$, fluorsianato de zinco $\left(\mathrm{F}_{6} \mathrm{SiZn} \cdot 6 \mathrm{H}_{2} \mathrm{O}\right)$ e dinitrofenol $\left[\left(\mathrm{NO}_{2}\right)_{2} \mathrm{C}_{6} \mathrm{H}_{3} \mathrm{OH}\right]$ se comportam de forma menos corrosiva, tendo a velocidade de formação de ferrugem em metais reduzida com o tempo.

O termo corrosão tem origem no latim corrodere (Mello, 2008; Nappi, 2012). A corrosão em metais ocorre pela destruição ou deterioração do material por causa de reações químicas e eletroquímicas, levando as ligas metálicas a retornarem a seu estado natural ou a um composto muito similar ao minério correspondente (Nappi, 2012).

A corrosão é um regresso a estados mais baixos de energia (energia de Gibbs), uma vez que o material metálico isolado está em um estado de energia mais elevado do que no minério correspondente e, em decorrência, em suas reações com o meio ambiente, esse material irá se combinar como óxidos, hidróxidos, carbonatos, silicatos, sulfetos e sulfatos, o que causa sua corrosão (Feliú \& Morcillo, 1982; Munger, 1999; Mello, 2008; Nappi, 2012).

Os processos de corrosão são espontâneos, contínuos e capazes de liberar energia, sendo considerados reações químicas heterogêneas ou eletroquímicas que ocorrem geralmente na interface entre o metal e o meio corrosivo (Feliú \& Morcillo, 1982).

A corrosão por reação pode ocorrer pelo contato com vapores ou gases, sem a presença de líquidos, normalmente esse tipo de corrosão está associado a altas temperaturas. Já uma reação eletroquímica ocorre em ambientes em que existem líquidos, por meio pulverizados ou imersos (Munger, 1999). A deterioração produz alterações prejudiciais indesejáveis no material, como desgaste, variações químicas ou modificações estruturais.

Carrasco (2009) afirma que entre as principais causas da corrosão de artefatos metálicos estão o oxigênio do ar, água ou umidade. O mecanismo que desencadeia a corrosão é acelerado pelo calor e pela presença de poluentes atmosféricos como dióxido de enxofre e dióxido de carbono, assim como pela presença de alguns sais.

Contudo, neste estudo enfatizam-se os processos de corrosão eletroquímica, que são mais frequentes na natureza e se caracterizam basicamente pela presença de água no estado líquido ou em temperaturas abaixo do ponto de orvalho da água. A maioria das corrosões ocorre à temperatura ambiente e com formação de pilha ou células de corrosão, com a circulação de elétrons na superfície metálica (Nappi, 2012).

Portanto, ao se considerar o emprego de materiais na construção de equipamentos ou instalações, além de eles possuírem propriedades mecânicas suficientes e características de fabricação adequadas, é necessário considerar que resistam à ação do meio corrosivo. A corrosão pode incidir sobre diversos tipos de materiais, sejam metálicos, como aços ou ligas de cobre, por exemplo, ou não metálicos, como plásticos, cerâmico ou concreto (Feliú \& Morcillo, 1982). O processo de corrosão apresenta grande importância do ponto de vista tecnológico e social, sendo objeto de muitos estudos (Nappi, 2012).

Paes et al. (2002a, b) citam que a determinação da corrosividade de um produto químico a metais pode ser feita em nível laboratorial ou em ensaios de campo. Os testes de laboratório podem fornecer importantes informações sobre a interação entre soluções salinas e equipamentos de plantas industriais madeireiras, como também sobre o efeito da madeira tratada em sobre metais empregados para sua fixação.

A corrosão do equipamento é simulada pela submersão de chapas limpas de metal em soluções preservativas. Após o período de teste, que normalmente é de 6 meses, as amostras são limpas e o grau de corrosão é avaliado pela perda de massa. Porém a American Society for Testing and Materials - ASTM D-130 (1991) preconiza a metodologia que prevê exposição de chapas de aço 
às soluções salinas nas temperaturas de $25^{\circ} \mathrm{C}, 45^{\circ} \mathrm{C}$ e $100{ }^{\circ} \mathrm{C}$ por 6 horas.

Para a indústria de construção civil, usinas de tratamento e câmaras de secagem de madeiras, a proteção contra corrosão é de grande importância técnica e econômica, já que a utilização de determinadas soluções salinas e preservativas em suas unidades fabris as pode inviabilizar. Diante do exposto, o estudo teve por objetivos avaliar a corrosividade de aço carbono por soluções salinas e o efeito delas após a sua impregnação em madeira.

\section{MATERIAL E MÉTODOS}

Para avaliar a corrosividade em metais foram seguidas as recomendações da ASTM D-130 (1991). Para tanto, foram preparadas soluções salinas com $5 \%$ de concentração, com base no sal seco, de cloreto de lítio, carbonato de sódio, sulfato de magnésio, sulfato de zinco e sulfato de cobre II. Conforme recomendações, as soluções foram mantidas em contato durante 6 horas nas temperaturas de $25^{\circ} \mathrm{C}, 45^{\circ} \mathrm{C}$ e $100{ }^{\circ} \mathrm{C}$ com amostras de $1,25 \mathrm{~cm} \times 0,1 \mathrm{~cm} \times 7,5 \mathrm{~cm}$ (largura $\times$ espessura $\times$ comprimento) confeccionadas a partir de chapas de aço classificadas pela Sociedade Automotiva de Engenheiros como SAE 1006, material empregado na produção de cilindros para as usinas de tratamento de madeira.

Soluções salinas preparadas de modo semelhante foram impregnadas em amostras de 2,0 $\mathrm{cm} \times 3,0 \mathrm{~cm} \times 5,0 \mathrm{~cm}$ (radial $\times$ tangencial $\times$ longitudinal) de madeira de cerne de Corymbia torelliana e Eucalyptus cloeziana com 15 anos de idade. Antes da impregnação, as amostras foram secas em estufa com circulação forçada, mantida a $103^{\circ} \mathrm{C} \pm 2{ }^{\circ} \mathrm{C}$, até massa constante, pesadas em balança com precisão de 0,01 g.
As soluções permaneceram em contato com as amostras até a completa saturação da madeira, o que foi determinado pela variação mínima da massa conforme estabelecido pela Norma Brasileira Regulamentadora - NBR 7190 da Associação Brasileira de Normas Técnicas, ABNT (1997).

Na comparação dos resultados da corrosividade das soluções salinas puras, empregou-se um delineamento inteiramente casualizado, com arranjo fatorial em que foram analisados os fatores: temperatura (3 níveis), solução salina (6 níveis) e a interação entre esses fatores. Já para avaliar o seu efeito após a impregnação da madeira, ao delineamento inicial proposto foi acrescido o fator espécie (2 níveis).

Os dados da perda de massa das chapas, em porcentagem, em virtude do efeito da corrosão, quando da necessidade para homogeneizar as variâncias, foram transformados em arcsen[raiz (perda de massa/100)], como recomendado por Steel \& Torrie (1980). Quando detectada diferença significativa entre os tratamentos, foi empregado o teste de Tukey, em nível de 5\% de significância, para a comparação entre as médias.

\section{RESULTADOS E DISCUSSÃO}

\subsection{Corrosividade das soluções salinas puras}

Observa-se na Tabela 1 a análise de variância para a perda de massa em porcentagem causada pela corrosividade das soluções de tratamento nas chapas de aço carbono (SAE 1006) em diferentes temperaturas, em que se verifica diferença significativa apenas entre as soluções salinas empregadas.

Dentre os efeitos corrosivos causados pelas soluções salinas, observou-se que a solução de sulfato de cobre II foi a que proporcionou maior perda de massa

Tabela 1. Análise de variância da perda de massa causada pelas soluções salinas em chapa de aço carbono (SAE 1006). Dados transformados em arcsen [raiz (perda de massa/100)].

Table 1. Analysis of variance of the mass loss caused for the saline solutions in steel carbon plate (SAE 1006). Data transformed into arcsen [root (mass loss/100)].

\begin{tabular}{lcccc}
\multicolumn{1}{c}{ Fonte de variação } & Graus de liberdade & Soma de quadrados & Quadrados médios & F \\
\hline Solução & 4 & 0,3033 & $0,76 \times 10^{-1}$ & $62,39^{* *}$ \\
Temperatura & 2 & $0,59 \times 10^{-2}$ & $0,29 \times 10^{-2}$ & $2,42^{\text {ns }}$ \\
Solução $\times$ Temperatura & 8 & $0,18 \times 10^{-1}$ & $0,22 \times 10^{-2}$ & $1,83^{\text {ns }}$ \\
Resíduo & 60 & $0,73 \times 10^{-1}$ & $0,12 \times 10^{-2}$ & \\
Total & $\mathbf{7 4}$ & $\mathbf{0 , 3 9 9 9}$ & & \\
\hline
\end{tabular}

** significativo a $1 \%$ de probabilidade; ns: não significativo a $5 \%$ de probabilidade. 
$(3,2974 \%)$, diferindo significativamente das demais (Tabela 2). Não houve perda de massa nas amostras controle, que permaneceram em contato com água destilada, não sendo incluídas na análise estatística.

Diversos autores, entre eles, Andersen et al. (1975), Jardy et al. (1992), Zelinsky et al. (2004), Rahmouni (2005) e Fitzgerald et al. (2006), ao estudarem a corrosividade de elementos químicos, constataram que soluções que continham cobre apresentaram maior corrosividade.

Em decorrência da corrosividade ocasionada pelo sultato de cobre, quando se deseja tratar a madeira por métodos sem pressão, como os de substituição de seiva e difusão, recomenda-se que a solução preparada com sais dessa natureza não seja acondicionada ou posta em contato com tambores metálicos.

Tabela 2. Perda de massa causada por corrosão pelas soluções salinas nas chapas de aço carbono (SAE 1006). Table 2. Mass loss caused for the corrosion of the saline solutions in steel carbon plates (SAE 1006).

\begin{tabular}{lc}
\multicolumn{1}{c}{ Soluções salinas } & Perda de massa (\%) \\
\hline Cloreto de lítio & $0,0065 \mathrm{~b}$ \\
\hline Carbonato de sódio & $0,0021 \mathrm{~b}$ \\
\hline Sulfato de magnésio & $0,0072 \mathrm{~b}$ \\
Sulfato de zinco & $0,0040 \mathrm{~b}$ \\
\hline Sulfato de cobre II & $3,2974 \mathrm{a}$ \\
\hline
\end{tabular}

Médias seguidas pela mesma letra minúscula não diferem estatisticamente entre si (Tukey, $\mathrm{p}>0,05$ ).

\subsection{Corrosividade das soluções salinas após sua impregnação na madeira}

A análise de variância da corrosividade causada pelas soluções salinas em chapas de aço carbono (SAE 1006), após a sua impregnação na madeira de Corymbia torelliana e de Eucalyptus cloeziana acusou diferença estatística para os fatores solução, espécie e temperatura e para a interação solução $\times$ temperatura de tratamento (Tabela 3). O efeito das espécies e a interação solução $\times$ temperatura foram analisados $e$ comparados pelo teste de média (Tabela 4).

Dentre os efeitos corrosivos causados pelas soluções em contato com a madeira, observou-se que a maior perda de massa ocorreu com as soluções empregadas para impregnar a madeira de Eucalyptus cloeziana, que diferiu estatisticamente do efeito causado pelo contato com a madeira de Corymbia torelliana.

O maior efeito corrosivo proporcionado pelas soluções que estiveram contato com a madeira de Eucalyptus cloeziana pode estar relacionado com o teor de extrativo da madeira. Paes et al. (2016), em trabalho desenvolvido com as mesmas madeiras, obteve valores de extrativos solúveis em etanol: tolueno $(2: 1 \mathrm{v} / \mathrm{v})$ de $4,65 \%$ e $5,40 \%$ para as madeira de Corymbia torelliana e Eucalyptus cloeziana, respectivamente.

Os resultados evidenciaram estatisticamente que as soluções contendo sulfato de zinco e sulfato de cobre II causaram maior perda de massa nas chapas de aço

Tabela 3. Análise de variância da perda de massa causada pelas soluções salinas em chapa de aço carbono (SAE 1006) após a impregnação da madeira de Corymbia torelliana e de Eucalyptus cloeziana. Dados transformados em arcsen [raiz (perda de massa/100)].

Table 3. Analysis of variance of the mass loss caused for the saline solutions in steel carbon plate (SAE 1006), after the impregnation of Corymbia torelliana and Eucalyptus cloeziana woods. Data transformed into arcsen [root (mass loss/100)].

\begin{tabular}{|c|c|c|c|c|}
\hline $\begin{array}{c}\text { Fontes de } \\
\text { variação }\end{array}$ & $\begin{array}{l}\text { Graus de } \\
\text { liberdade }\end{array}$ & $\begin{array}{c}\text { Soma de } \\
\text { quadrados }\end{array}$ & $\begin{array}{l}\text { Quadrados } \\
\text { médios }\end{array}$ & $\mathbf{F}$ \\
\hline Solução & 2 & $0,17 \times 10^{-1}$ & $0,83 \times 10^{-2}$ & $126,16^{\star *}$ \\
\hline Espécie & 1 & $0,22 \times 10^{-2}$ & $0,22 \times 10^{-2}$ & $9,15^{\star *}$ \\
\hline Temperatura & 2 & $0,17 \times 10^{-1}$ & $0,83 \times 10^{-2}$ & $34,11^{\star *}$ \\
\hline Solução $\times$ Espécie & 5 & $0,11 \times 10^{-2}$ & $0,22 \times 10^{-3}$ & $0,88^{\mathrm{ns}}$ \\
\hline Solução $\times$ Temperatura & 10 & $0,36 \times 10^{-1}$ & $0,36 \times 10^{-3}$ & $14,75^{\star *}$ \\
\hline Espécie $\times$ Temperatura & 2 & $0,12 \times 10^{-2}$ & $0,59 \times 10^{-3}$ & $2,40^{\text {ns }}$ \\
\hline Temperatura $\times$ Espécie $\mathrm{x}$ Solução & 10 & $0,25 \times 10^{-2}$ & $0,25 \times 10^{-3}$ & $1,02^{\mathrm{ns}}$ \\
\hline Resíduo & 144 & 27,5552 & 0,1914 & \\
\hline Total & 179 & 0,2489 & & \\
\hline
\end{tabular}

** significativo a $1 \%$ de probabilidade; ns: não significativo a $5 \%$ de probabilidade. 
Tabela 4. Perda de massa causada por corrosão pelas soluções salinas em chapas de aço carbono (SAE 1006) após a impregnação da madeira de Corymbia torelliana e de Eucalyptus cloeziana.

Table 4. Mass loss caused for the corrosion of the saline solutions in steel carbon plates (SAE 1006), after the impregnation of Corymbia torelliana and Eucalyptus cloeziana woods.

\begin{tabular}{|c|c|c|c|}
\hline \multirow{3}{*}{ Espécie } & Corymbia torelliana & & Eucalyptus cloeziana \\
\hline & Perda de massa (\%) & & \\
\hline & $0,1910 \mathrm{~B}$ & & $0,2302 \mathrm{~A}$ \\
\hline \multirow{3}{*}{ Solução salina } & Temperatura $\left({ }^{\circ} \mathrm{C}\right)$ & & \\
\hline & 25 & 45 & 100 \\
\hline & Perda de massa (\%) & & \\
\hline Água destilada & $0,0127 \mathrm{Ab}$ & $0,0217 \mathrm{Ab}$ & $0,0557 \mathrm{Abc}$ \\
\hline Cloreto de lítio & $0,0299 \mathrm{Ab}$ & $0,0351 \mathrm{Ab}$ & $0,0379 \mathrm{Abc}$ \\
\hline Carbonato de sódio & $0,0052 \mathrm{Ab}$ & $0,0043 \mathrm{Ab}$ & $0,0079 \mathrm{Ac}$ \\
\hline Sulfato de magnésio & $0,0094 \mathrm{Ab}$ & $0,0199 \mathrm{Ab}$ & $0,0446 \mathrm{Abc}$ \\
\hline Sulfato de zinco & $0,0130 \mathrm{Bb}$ & $0,0218 \mathrm{ABb}$ & $0,1011 \mathrm{Ab}$ \\
\hline Sulfato de cobre II & $0,4333 \mathrm{Ba}$ & $0,4911 \mathrm{Ba}$ & $2,4458 \mathrm{Aa}$ \\
\hline
\end{tabular}

carbono (SAE 1006), o que as diferenciou das demais soluções empregadas. Paes et al. (2002b), ao avaliarem a corrosividade de soluções preservativas preparadas com creosoto vegetal, observaram que soluções que continham cobre causaram maior corrosão.

Nota-se ainda que o nível de corrosão do sulfato de zinco foi estatisticamente igual para as temperaturas de $25^{\circ} \mathrm{C}, 45^{\circ} \mathrm{C}$ e $100^{\circ} \mathrm{C}$ (Tabela 4 ). Nas temperaturas $25^{\circ} \mathrm{C}$ e $45^{\circ} \mathrm{C}$ observa-se que somente a solução de sulfato de cobre II causou perda de massa significativa quando comparada com a das demais soluções.

Observou-se que na temperatura de $100{ }^{\circ} \mathrm{C}$ somente a solução de sulfato de cobre II causou perda de massa significativa, o que a diferenciou das demais soluções empregadas. Nota-se ainda que as soluções de cloreto de lítio, sulfato de magnésio e sulfato de zinco causaram efeito corrosivo semelhante ao controle (água destilada). Já nesse quesito a solução de carbonato de sódio diferiu estatisticamente das soluções de sulfato de zinco e sulfato de cobre II (Tabela 4).

Ao se avaliar o efeito da temperatura, notou-se que as soluções sulfato de zinco e sulfato de cobre II não devem ser indicadas para a impregnação de madeira em instalações industriais. Segundo Paes et al. (2002b), o aumento da corrosividade com a temperatura do tratamento poderia por em risco equipamentos da usina de tratamento da madeira, bem como a integridade física dos operários.

O maior valor de corrosão causado pela solução de sulfato de cobre II (Tabela 2), quando comparado com os apresentados na Tabela 4, pode ser explicado pela absorção do sal pela madeira. Isso foi observado por Paes et al. (2015) ao submeter tais amostras a secagem em estufa.

\section{CONCLUSÕES}

A solução de sulfato de cobre II apresentou a maior taxa de corrosão, não sendo indicado seu contato com instalações metálicas produzidas com aço carbono.

As soluções salinas empregadas para impregnar a madeira de Eucalyptus cloeziana causaram maior corrosividade nas chapas de aço carbono.

A temperatura influenciou na corrosividade apenas para as soluções sulfato de zinco e sulfato de cobre II quando em contato com a madeira empregada.

\section{STATUS DA SUBMISSÃO}

Recebido: 7 nov., 2014

Aceito: 26 jun., 2016

\section{AUTOR(ES) PARA CORRESPONDÊNCIA}

\section{Juarez Benigno Paes}

Departamento de Ciências Florestais e da Madeira, Universidade Federal do Espirito Santo - UFES, Av. Gobernador Lindember, 316, Centro, CEP 29500-000, Jeronimo Monteiro, ES, Brasil e-mail: jbp2@uol.com.br 


\section{REFERÊNCIAS}

American Society for Testing and Materials - ASTM. D-130: standard test method for detection of copper corrosion from petroleum products by the copper strip tarnish test. In: ASTM. Annual Book of ASTM Standards. West Conshohocken; 1991. vol. 0501. p. 79-84.

Andersen TN, Ghandehari MH, Eyiring H. A limitation to the mixed potential concept of metal corrosion. Journal of the Electrochemical Society 1975; 122(12): 1580-1585. http://dx.doi.org/10.1149/1.2134073.

Associação Brasileira de Normas Técnicas - ABNT. NBR 7190: projeto de estruturas de madeira. Rio de Janeiro; 1997. $107 \mathrm{p}$.

Carrasco GLA. Preservação de artefatos ornamentais de ferro integrados à arquitetura - estudo de caso: cemitério do imigrante, Joinville, SC [dissertação]. Florianópolis: Universidade Federal de Santa Catarina; 2009. 133 p.

Feliú S, Morcillo M. Corrosión y protección de los metales en la atmósfera. Madrid: Ediciones Bellaterra S. A.; 1982. $246 \mathrm{p}$.

Fitzgerald KP, Nairn J, Skennerton G, Atrens A. Atmospheric corrosion of copper and the colour, structure and composition of natural patinas on copper. Corrosion Science 2006; 48(9): 2480-2509. http://dx.doi.org/10.1016/j. corsci.2005.09.011.

Jardy A, Lasalle-Molin AL, Keddam M, Takenoutti H. Copper dissolution in acidic sulphate media studied by QCM and RRDE under AC signal. Electrochimica Acta 1992; 37(12): 2195-2201. http://dx.doi.org/10.1016/00134686(92)85111-W.

Kollmann F. Tecnologia de la madeira y sus aplicaciones. Madrid: Gráficas Reunidas S.A.; 1959. 675 p.

Kollmann FFP, Côté, WA Jr. Principles of wood science and technology. New York: Springer-Verlag; 1968. vol. 1, 592 p.

Mello AGB. Influência da deformação plástica na corrosão de aço carbono, aço inoxidável e cobre em sistemas de resfriamento [dissertação]. Rio de Janeiro: Universidade Federal do Rio de Janeiro; 2008. 79 p.

Munger CG. Corrosion prevention by protective coatings. 2nd ed. Houston: National Association of Corrosion Engineers; 1999.520 p.

Nappi MML. Corrosão de elementos metálicos embutidos em diferentes espécies de madeira [dissertação]. Florianópolis: Universidade Federal de Santa Catarina; 2012. 233 p.

Paes JB, Guerra SCS, Silva LF, Oliveira JGL, Teago GBS. Efeito do teor de extrativos na resistência natural de cinco madeiras ao ataque de cupins xilófagos. Ciência Florestal 2016; 26(4): 1259-1269. http://dx.doi.org/10.5902/1980509825137.

Paes JB, Loiola PL, Oliveira JTS, Braz RL, Klitzke RJ. Efeito de soluções salinas na estabilidade dimensional das madeiras de Corymbia torelliana e Eucalyptus cloeziana. Scientia Forestal 2015; 43(106): 271-281.

Paes JB, Vital BR, Della Lucia RM, Della Lucia TMC. Corrosão de parafusos fixados à madeira tratada com soluções de creosoto vegetal. Revista Árvore 2002a; 26(3): 357-361. http://dx.doi.org/10.1590/S0100-67622002000300011.

Paes JB, Vital BR, Della Lucia RM, Della Lucia TMC. Corrosividade causada por soluções produzidas com creosoto vegetal. Revista Árvore 2002b; 26(5): 621-627. http://dx.doi.org/10.1590/S0100-67622002000500013.

Rahmouni K. Corrosion et protection des bronzes recouverts de patine: étude eletrochimique et spectroscopique de la surface d'objets archeologiques et synthese d'une patine equivalente sur un bronze du commerce [thèse]. Paris: Université Pierre et Marie Curie; Marrocos: Université IBN Tofail; 2005. 223 p

Steel RGD, Torrie JH. Principles and procedures of statistic: a biometrical approach. 2nd ed. New York: McGraw-Hill; $1980.633 \mathrm{p}$.

Zelinsky AG, Pirogov BY, Yurjev OA. Open circuit potential transients and electrochemical quartz crystal micro gravimetry measurements of dissolution of copper in acidic sulfate solutions. Corrosion Science 2004; 46(5): 1083-1093. http://dx.doi.org/10.1016/j.corsci.2003.09.008. 